Pacific Journal of Mathematics

SPECTRAL SETS AS BANACH MANIFOLDS

A Gel RAFAEL LAROTONDA AND IGNACIO ZALDUENDO 


\title{
SPECTRAL SETS AS BANACH MANIFOLDS
}

\author{
ANGEL LAROTONDA AND IGNACIO ZALDUENDO
}

Let $A$ be a commutative Banach algebra, $X$ its spectrum, and $M$ a closed analytic submanifold of an open set in $C^{n}$. We may consider the set of germs of holomorphic functions from $X$ to $M, \mathcal{O}(X, M)$. Now let $\nu$ be the functional calculus homomorphism from $\mathcal{O}\left(X, C^{n}\right)$ to $A^{n}$, and $A_{M}=\nu(\mathcal{O}(X, M))$.

It is proven that $A_{M}$ is an analytic submanifold of $A^{n}$, modeled on projective $A$-modules of $\operatorname{rank}=\operatorname{dim} M$.

1. Introduction. Let $A$ be a commutative complex Banach algebra with identity, and let $X$ be the set of all characters of $A$, considered as a compact subset of the topological dual $A^{\prime}$ with the weak*-topology.

If $U$ is an open neighborhood of $X$, and $B$ a complex Banach space a map $f: U \rightarrow B$ will be called holomorphic if it is locally bounded and all its complex directional derivatives exist. The set of all such functions which are also bounded on $U$ will be denoted by $H^{\infty}(U, B)$, or simply $H^{\infty}(U)$, when $B$ is the complex field. These are locally convex spaces with the topology of uniform convergence. We define $\mathcal{O}(X, B)$ and $\mathcal{O}(X)$ to be the inductive limit of these spaces as $U$ ranges over all open neighborhoods of $X . \mathcal{O}(X)$ is then a topological algebra. We recall (see [2] or [7]) that there exists a continuous algebra epimorphism, the holomorphic functional calculus

$$
\nu: \mathcal{O}(X) \rightarrow A
$$

such that: the composition of $\nu$ and the Gelfand map

$$
\mathcal{O}(X) \rightarrow A \rightarrow C(X)
$$

is the restriction map $\left.f \mapsto f\right|_{X}$, and the composition of the linear map $a \mapsto \tilde{a}$ and $\nu$

$$
A \rightarrow \mathcal{O}(X) \rightarrow A
$$

is the identity map of $A$. Here $\tilde{a}$ denotes the germ of the holomorphic map defined on $A^{\prime}$ by $\gamma \mapsto \gamma(a)$.

In [6], Raeburn has generalized previous results of Taylor and Novodvorskii ([7],[5]). He uses a generalization of the morphism $\nu$, extending the holomorphic functional calculus to a linear map

$$
S: \mathcal{O}(X, B) \rightarrow A \hat{\otimes} B .
$$


If $M \subset B$ denotes a Banach submanifold, $\mathcal{O}(X, M)$ is defined and so is the set

$$
A_{M}=\{S(f): f \in \mathcal{O}(X, M)\} \subset A \hat{\otimes} B .
$$

Raeburn shows that if $M$ is a discrete union of Banach homogeneous spaces the set $A_{M}$ is locally path connected and the generalized Gelfand map

$$
A_{M} \rightarrow C(X, M)
$$

induces a bijection on the set of components

$$
\left[A_{M}\right] \stackrel{\simeq}{\rightarrow}[X, M] \text {. }
$$

In this note, in $\S 3$, we take $B=\mathbf{C}^{n}$ and $M$ a closed submanifold of an open set of $\mathbf{C}^{n}$, and prove that the set $A_{M}$ is in fact an analytic submanifold of $A^{n}$. This was first stated by Taylor in [8]. $A_{M}$ is modeled on projective $A$-modules of rank $=\operatorname{dim} M$. We also prove that $A_{M}$ and $A^{M}=\left\{a \in A^{n}: \operatorname{sp}(a) \subset M\right\}$ have the same homotopy type. Note that with $B=\mathbf{C}^{n}$, we have $S=\nu \times \cdots \times \nu$ and $A \hat{\otimes} B=A^{n}$.

In order to do this we first prove in $\S 2$ a version of the constant rank theorem.

2. The constant rank theorem. In this paragraph we give a version of the constant rank theorem valid for $A$-modules; the whole paragraph is an adaptation of the results in [4].

We will be dealing with submodules of the free module $A^{n}$, and $A$-module morphisms $T: A^{n} \rightarrow A^{m}$. A submodule $E$ of $A^{n}$ will be called $A$-direct if it is closed and there is another closed submodule $E^{\prime}$ of $A^{n}$ such that $A^{n}=E \oplus E^{\prime}$; obviously, this is equivalent to the fact: $E=\operatorname{Ker} p$ (resp: $E=\operatorname{Im} p$ ), for some continuous $A$-linear projector $p: A^{n} \rightarrow A^{n}$.

Note that in this case $E$ is a projective module, but not necessarily free.

If $T: A^{n} \rightarrow A^{m}$ is an $A$-module morphism, we say that $T$ is $A$-direct (also called "split") if $\operatorname{Ker} T$ and $\operatorname{Im} T$ are $A$-direct.

Assume that

$$
A^{n}=E_{1} \oplus E_{2}, \quad F_{1} \oplus F_{2}=A^{m}
$$

for some closed submodules $E_{1}, E_{2}, F_{1}, F_{2}$; if $T: A^{n} \rightarrow A^{m}$ is an $A$-morphism we shall use the notation

$$
T=\left[\begin{array}{ll}
T_{11} & T_{12} \\
T_{21} & T_{22}
\end{array}\right]:\left[\begin{array}{l}
E_{1} \\
E_{2}
\end{array}\right] \rightarrow\left[\begin{array}{l}
F_{1} \\
F_{2}
\end{array}\right]
$$


with $T_{i j} \in \operatorname{Hom}_{A}\left(E_{j}, F_{l}\right)(i, j=1,2)$, meaning that if

$$
x=x_{1}+x_{2} \quad\left(x_{1} \in E_{1}, x_{2} \in E_{2}\right),
$$

then

$$
T(x)=\left[T_{11}\left(x_{1}\right)+T_{12}\left(x_{2}\right)\right]+\left[T_{21}\left(x_{1}\right)+T_{22}\left(x_{2}\right)\right]
$$

is the expression of $T(x)$ as a sum of elements in $F_{1}$ and $F_{2}$.

We shall need the following elementary lemma, which we state without proof.

LeMma 2.1. Let $P_{1}, P_{2}$ be $A$-direct submodules of $A^{n}$ of the same rank. Then $P_{1} \subset P_{2}$ implies $P_{1}=P_{2}$.

THEOREM 1. Suppose $T_{0}: A^{n} \rightarrow A^{m}$ is an A-direct morphism and let $E_{1}$ and $F_{2}$ be closed submodules of $A^{n}$ and $A^{m}$ respectively such that

$$
A^{n}=E_{1} \oplus \operatorname{Ker} T_{0}, \quad \operatorname{Im} T_{0} \oplus F_{2}=A^{m}
$$

If

$$
T=\left[\begin{array}{ll}
\alpha & \beta \\
\gamma & \delta
\end{array}\right]:\left[\begin{array}{c}
E_{1} \\
\operatorname{Ker} T_{0}
\end{array}\right] \rightarrow\left[\begin{array}{c}
\operatorname{Im} T_{0} \\
F_{2}
\end{array}\right]
$$

then the following are equivalent

(i) $T$ is $A$-direct, $A^{n}=E_{1} \oplus \operatorname{Ker} T$ and $A^{m}=\operatorname{Im} T \oplus F_{2}$.

(ii) $\alpha \in \operatorname{Iso}\left(E_{1}, \operatorname{Im} T_{0}\right)$ and $\delta=\gamma \alpha^{-1} \beta$.

(iii) There exist $A$-linear automorphisms $u: A^{n} \rightarrow A^{n}, v: A^{m} \rightarrow A^{m}$ such that $T_{0}=v T u$ and

$$
u\left|E_{1}=\mathrm{id}_{E_{1}} \quad v\right| F_{2}=\mathrm{id}_{F_{2}} .
$$

(iv) $T$ is $A$-direct, $\alpha \in \operatorname{Iso}\left(E_{1}, \operatorname{Im} T_{0}\right)$ and $\operatorname{rk}\left(\operatorname{Im} T_{0}\right)=\operatorname{rk}(\operatorname{Im} T)$.

Proof: Suppose (i) and consider the diagram

$$
\begin{array}{ccc}
E_{1} \times \operatorname{Ker} T & \stackrel{w}{\rightarrow} & \operatorname{Im} T \times F_{2} \\
\phi \uparrow & & \downarrow \psi \\
A^{n}=E_{1} \oplus \operatorname{Ker} T_{0} & \stackrel{T}{\rightarrow} & \operatorname{Im} T_{0} \oplus F_{2}=A^{m}
\end{array}
$$

where $\phi$ is the isomorphism $v \rightarrow\left(v_{1}, v_{2}\right)$; here $v_{1}$ (resp: $\left.v_{2}\right)$ is the projection of $v$ onto $E_{1}$ (resp: $\operatorname{Ker} T$ ) with kernel $\operatorname{Ker} T$ (resp. $E_{1}$ ). We define $\psi$ 
in a similar way. Then we have

$$
\phi=\left[\begin{array}{ll}
1 & \tau \\
0 & \theta
\end{array}\right]:\left[\begin{array}{c}
E_{1} \\
\operatorname{Ker} T_{0}
\end{array}\right] \rightarrow\left[\begin{array}{c}
E_{1} \\
\operatorname{Ker} T
\end{array}\right]
$$

and

$$
\psi=\left[\begin{array}{ll}
\mu & 0 \\
\nu & 1
\end{array}\right]:\left[\begin{array}{c}
\operatorname{Im} T \\
F_{2}
\end{array}\right] \rightarrow\left[\begin{array}{c}
\operatorname{Im} T_{0} \\
F_{2}
\end{array}\right]
$$

with $\tau \in \operatorname{Hom}_{A}\left(\operatorname{Ker} T_{0}, E_{1}\right), \quad \nu \in \operatorname{Hom}_{A}\left(\operatorname{Im} T, F_{2}\right)$ and $\theta \in$ $\mathrm{Iso}_{A}\left(\operatorname{Ker} T_{0}, \operatorname{Ker} T\right), \mu \in \operatorname{Iso}_{A}\left(\operatorname{Im} T, \operatorname{Im} T_{0}\right)$. On the other hand we also have

$$
w=\left[\begin{array}{ll}
\lambda & 0 \\
0 & 0
\end{array}\right]:\left[\begin{array}{c}
E_{1} \\
\operatorname{Ker} T
\end{array}\right] \rightarrow\left[\begin{array}{c}
\operatorname{Im} T \\
F_{2}
\end{array}\right]
$$

with $\lambda \in \operatorname{Iso}_{A}\left(E_{1}, \operatorname{Im} T\right)$.

The commutativity of the diagram implies

$$
\left[\begin{array}{ll}
\mu & 0 \\
\nu & 1
\end{array}\right]\left[\begin{array}{ll}
\lambda & 0 \\
0 & 0
\end{array}\right]\left[\begin{array}{ll}
1 & \tau \\
0 & \theta
\end{array}\right]=\left[\begin{array}{ll}
\alpha & \beta \\
\gamma & \delta
\end{array}\right],
$$

hence $\mu \lambda=\alpha$ (which implies that $\alpha$ is an isomorphism) and $\delta=\nu \lambda \tau=$ $\nu \lambda\left(\lambda^{-1} \mu^{-1}\right) \mu \lambda \tau=\gamma \alpha^{-1} \beta$, and we have (ii). Now assume (ii): if

$$
T_{0}=\left[\begin{array}{ll}
\lambda & 0 \\
0 & 0
\end{array}\right]:\left[\begin{array}{c}
E_{1} \\
\operatorname{Ker} T_{0}
\end{array}\right] \rightarrow\left[\begin{array}{c}
\operatorname{Im} T_{0} \\
F_{2}
\end{array}\right]
$$

with $\lambda \in \operatorname{Iso}_{A}\left(E_{1}, \operatorname{Im} T_{0}\right)$ we define

$$
u=\left[\begin{array}{cc}
1 & -\alpha^{-1} \beta \\
0 & 1
\end{array}\right]:\left[\begin{array}{c}
E_{1} \\
\operatorname{Ker} T_{0}
\end{array}\right] \rightarrow\left[\begin{array}{c}
E_{1} \\
\operatorname{Ker} T_{0}
\end{array}\right]
$$

and

$$
v=\left[\begin{array}{cc}
\lambda \alpha^{-1} & 0 \\
-\gamma \alpha^{-1} & 1
\end{array}\right]:\left[\begin{array}{c}
\operatorname{Im} T_{0} \\
F_{2}
\end{array}\right] \rightarrow\left[\begin{array}{c}
\operatorname{Im} T_{0} \\
F_{2}
\end{array}\right]
$$

and a routine calculation gives (iii).

Now suppose we have (iv) and define the automorphism $S: A^{m} \rightarrow A^{m}$ by

$$
S=\left[\begin{array}{cc}
1 & 0 \\
-\gamma \alpha^{-1} & 1
\end{array}\right]:\left[\begin{array}{c}
\operatorname{Im} T_{0} \\
F_{2}
\end{array}\right] \rightarrow\left[\begin{array}{c}
\operatorname{Im} T_{0} \\
F_{2}
\end{array}\right]
$$


Then we have the composition

$$
T^{\prime}=S T=\left[\begin{array}{cc}
\alpha & \beta \\
0 & \delta-\gamma \alpha^{-1} \beta
\end{array}\right]:\left[\begin{array}{c}
E_{1} \\
\operatorname{Ker} T_{0}
\end{array}\right] \rightarrow\left[\begin{array}{c}
\operatorname{Im} T_{0} \\
F_{2}
\end{array}\right]
$$

which is also $A$-direct. Note that $\operatorname{Im}\left(T^{\prime}\right)=S(\operatorname{Im} T)$, hence $\operatorname{Im}\left(T^{\prime}\right)$ and $\operatorname{Im}(T)$ have the same rank; from this it follows that $\mathrm{rk}\left(\operatorname{Im} T^{\prime}\right)=\operatorname{rk}\left(\operatorname{Im} T_{0}\right)$.

But $\operatorname{Im}\left(T^{\prime}\right) \supset \alpha\left(E_{1}\right)=\operatorname{Im}\left(T_{0}\right) ;$ Lemma 2.1 gives $\operatorname{Im}\left(T^{\prime}\right)=\operatorname{Im}\left(T_{0}\right)$ and this fact implies $\delta-\gamma \alpha^{-1} \beta=0$. This proves (ii)

(iii) $\Rightarrow$ (i) is simple; in fact, it is obvious that $T$ is $A$-direct. It is also clear that $u\left(\operatorname{Ker} T_{0}\right)=\operatorname{Ker} T$, hence

$$
\begin{aligned}
A^{m} & =v^{-1}\left(\operatorname{Im} T_{0} \oplus F_{2}\right)=v^{-1}\left(\operatorname{Im} T_{0}\right) \oplus v^{-1}\left(F_{2}\right) \\
& =v^{-1} T_{0}\left(A^{n}\right) \oplus F_{2}=T u\left(A^{n}\right) \oplus F_{2}=\operatorname{Im} T \oplus F_{2}, \\
A^{n} & =u\left(\operatorname{Ker} T_{0} \oplus E_{1}\right)=u\left(\operatorname{Ker} T_{0}\right) \oplus E_{1}=\operatorname{Ker} T \oplus E_{1} .
\end{aligned}
$$

In order to complete the proof, we only need the inference (i) $\Rightarrow$ (iv): $\alpha \in \operatorname{Iso}\left(E_{1}, \operatorname{Im} T_{0}\right)$ as in (i) $\Rightarrow$ (ii). The rest is obvious, so the proof is complete.

We shall be concerned now with a generalization of the results in $\S 1$ of [6], we shall follow the definitions of this reference.

Let $\Omega$ be an open set in $A^{n}, F: \Omega \rightarrow A^{m}$ an holomorphic map, and $a \in \Omega$; we denote the differential of $F$ at $a$ by $D F(a)$.

A linear representation of $F$ in $a$ is an object $(u, U, v, V, T)$ where

(i) $U$ is a neighborhood of $0 \in A^{n}, u$ is biholomorphic from $U$ onto $u(U)$, a neighborhood of $a$ contained in $\Omega$, and $u(0)=a$.

(ii) $V$ is a neighborhood of $0 \in A^{m}, v$ is biholomorphic from $V$ onto $v(V)$, a neighborhood of $F(a)$ and $v(0)=F(a)$

(iii) $T: U \rightarrow A^{m}$ is the restriction of an $A$-linear map, and $v^{-1} F u=T$.

(iv) $D u(x)$ and $D v(y)$ are $A$-linear maps if $x \in U, y \in V$.

We will say that the holomorphic map $F: \Omega \rightarrow A^{m}$ is locally $A$-direct at $a \in \Omega$ if there are closed sub-modules $E_{1} \subset A^{n}, F_{2} \subset A^{m}$ and a neighborhood $U$ of $a$ such that, for all $x \in U$,

(i) $D F(x)$ is $A$-linear

(ii) $A^{n}=E_{1} \oplus \operatorname{Ker} D F(x)$

(iii) $A^{m}=\operatorname{Im} D F(x) \oplus F_{2}$.

We have now the following:

Lemma 2.2. Let $\Omega$ be an open set in $A^{n}, F: \Omega \rightarrow A^{m}$ holomorphic and $a \in \Omega$. If $F$ is locally $A$-direct at a, then there is a linear representation ( $u, U, v, V, T)$ of $F$ in a, with $T A$-direct. 
Proof. Without loss of generality we can assume that $a=0$ and $F(a)=0$; then there exist a neighborhood $\Omega_{0} \subset \Omega$ of $0 \in A^{n}$ and closed submodules $E_{1} \subset A^{n}, F_{2} \subset A^{m}$ such that

$$
A^{n}=E_{1} \oplus \operatorname{Ker} D F(x), \quad A^{m}=\operatorname{Im} D F(x) \oplus F_{2}
$$

for all $x \in \Omega_{0}$. Also, $D F(x)$ is $A$-linear if $x \in \Omega_{0}$.

Let $E_{2}=\operatorname{Ker} D F(0), F_{1}=\operatorname{Im} D F(0)$; we denote $x_{1}, x_{2}$ (resp: $\left.y_{1}, y_{2}\right)$ the components of $x \in A^{n}$ (resp: $y \in A^{m}$ ) in the decomposition $E_{1} \oplus E_{2}$ (resp: $F_{1} \oplus F_{2}$ ). In a similar way we write $F(x)=f_{1}(x)+f_{2}(x)$, with $f_{1}(x) \in F_{1}$ and $f_{2}(x) \in F_{2}$.

We have

$$
D F(x)=\left[\begin{array}{ll}
D_{1} f_{1}(x) & D_{2} f_{1}(x) \\
D_{1} f_{2}(x) & D_{2} f_{2}(x)
\end{array}\right]:\left[\begin{array}{l}
E_{1} \\
E_{2}
\end{array}\right] \rightarrow\left[\begin{array}{l}
F_{1} \\
F_{2}
\end{array}\right]
$$

and so we can simplify the notation writing $\alpha_{i j}(x)=D_{i} f_{j}(x)(i, j=1,2)$. Recall that Theorem 1 gives

(a) $\alpha_{11}(x): E_{1} \rightarrow F_{1}$ is an isomorphism, and

(b) $\alpha_{22}(x)=\alpha_{12}(x) \alpha_{11}(x)^{-1} \alpha_{21}(x)$ for all $x \in \Omega_{0}$.

Define the following $A$-linear maps

$$
\begin{array}{ll}
S: E_{1} \rightarrow F_{1}, & S=\alpha_{11}(0), \\
T: A^{n} \rightarrow A^{m}, & T(x)=S\left(x_{1}\right), \\
c: A^{m} \rightarrow A^{n}, & c(y)=S^{-1}\left(y_{1}\right), \\
P: A^{n} \rightarrow A^{n}, & P(x)=x_{2}, \\
Q: A^{m} \rightarrow A^{m}, & Q(y)=y_{2} .
\end{array}
$$

Now define the holomorphic map $h: \Omega_{0} \rightarrow A^{n}$ by

$$
h=c F+P .
$$

We have: $D h(x)$ is an $A$-linear map if $x \in \Omega_{0}$. In fact,

$$
\begin{aligned}
D h(x) & =\left[\begin{array}{cc}
S^{-1} & 0 \\
0 & 0
\end{array}\right]\left[\begin{array}{cc}
\alpha_{11}(x) & \alpha_{21}(x) \\
\alpha_{12}(x) & \alpha_{22}(x)
\end{array}\right]+\left[\begin{array}{ll}
0 & 0 \\
0 & 1
\end{array}\right] \\
& =\left[\begin{array}{cc}
S^{-1} \alpha_{11}(x) & S^{-1} \alpha_{21}(x) \\
0 & 1
\end{array}\right]
\end{aligned}
$$

hence by the inverse function theorem $h: \Omega_{1} \rightarrow \Omega_{2}$ is biholomorphic for suitable neighborhoods of $0 \in A^{n}$.

Note that the differential of the map $F h^{-1} P: P^{-1}\left(\Omega_{2}\right) \rightarrow A^{m}$ vanishes identically, that is

$$
D\left(F h^{-1} P\right)(x)=0 \quad\left(x \in P^{-1}\left(\Omega_{2}\right)\right) .
$$


In fact we can compute this differential as the composition $D F\left(h^{-1} P(x)\right) D h\left(h^{-1} P(x)\right)^{-1} P$; the calculation leads (with $x^{\prime}=h^{-1} P(x)$ ) to

$$
\begin{gathered}
{\left[\begin{array}{cc}
\alpha_{11}\left(x^{\prime}\right) & \alpha_{21}\left(x^{\prime}\right) \\
\alpha_{12}\left(x^{\prime}\right) & \alpha_{22}\left(x^{\prime}\right)
\end{array}\right]\left[\begin{array}{cc}
\alpha_{11}\left(x^{\prime}\right)^{-1} S & -\alpha_{11}\left(x^{\prime}\right)^{-1} \alpha_{21}(x) \\
0 & 1
\end{array}\right]\left[\begin{array}{ll}
0 & 0 \\
0 & 1
\end{array}\right]} \\
=\left[\begin{array}{cc}
S & 0 \\
\alpha_{12}\left(x^{\prime}\right) \alpha_{11}\left(x^{\prime}\right)^{-1} S & 0
\end{array}\right]\left[\begin{array}{ll}
0 & 0 \\
0 & 1
\end{array}\right]=0,
\end{gathered}
$$

where we use the identity $\alpha_{22}=\alpha_{12} \alpha_{11}^{-1} \alpha_{21}$.

Hence we have proved

(c) $F h^{-1} P$ vanishes identically in a neighborhood of 0 (for instance, in the connected component of 0 in $P^{-1}\left(\Omega_{2}\right)$ ).

Finally we define the holomorphic mapping $g: c^{-1}\left(\Omega_{2}\right) \rightarrow A^{m}$

$$
g=F h^{-1} c+Q \text {. }
$$

Then if $x=h^{-1} c(y)$ we compute

$$
D g(y)=\left[\begin{array}{cc}
1 & 0 \\
\alpha_{12}(x) \alpha_{11}(x)^{-1} & 1
\end{array}\right]
$$

and this shows that $g: \Omega_{1}{ }^{\prime} \rightarrow \Omega_{2}{ }^{\prime}$ is a biholomorphic map, where $\Omega_{1}^{\prime}$ and $\Omega_{2}^{\prime}$ are small enough neighborhoods of $0 \in A^{m}$. Also $D g(y)$ is $A$-linear for every $x \in \Omega_{1}{ }^{\prime}$.

In order to complete the proof, set $u=h^{-1}$ and $v=g$; we must show that the identity

$$
g T h=F
$$

holds in some neighborhood of $0 \in A^{n}$; but this follows from (c) and the computation

$$
\begin{aligned}
g T h & =\left(F h^{-1} c+Q\right) T(c F+P)=F h^{-1} c Q F \\
& =F h^{-1} c F=F h^{-1}(h-P)=F-F h^{-1} P .
\end{aligned}
$$

THEOREM 2. Let $\Omega$ be an open subset of $A^{n}$, and $F: \Omega \rightarrow A^{n}$ an holomorphic retraction that is locally $A$-direct at $x$ for all $x \in \Omega$. Then $\operatorname{Im} F$ is a Banach analytic manifold, and for all $x \in \operatorname{Im} F$ the tangent space $T_{x}(\operatorname{Im} F)$ at $x$ is $\operatorname{Im} D F(x)$.

Proof. For every $F(x) \in \operatorname{Im} F$ there is, by Lemma 2.2, a linear representation $\left(u_{x}, U_{x}, v_{x}, V_{x}, T_{x}\right)$ of $F$ with $T_{x} A$-direct. 
For all $x^{\prime} \in U_{x}$,

$$
\begin{aligned}
T_{x} & =D T_{x}\left(x^{\prime}\right)=D v_{x}^{-1}\left(F u_{x}\left(x^{\prime}\right)\right) \cdot D F\left(u_{x}\left(x^{\prime}\right)\right) \cdot D u_{x}\left(x^{\prime}\right) \\
& =\left[D v_{x}\left(T_{x}\left(x^{\prime}\right)\right)\right]^{-1} \cdot D F\left(u_{x}\left(x^{\prime}\right)\right) \cdot D u_{x}\left(x^{\prime}\right) .
\end{aligned}
$$

$D v_{x}(Z)$ and $D u_{x}\left(Z^{\prime}\right)$ are $A$-linear isomorphisms, so $\operatorname{Im} T_{x} \simeq$ Im $D F\left(u_{x}\left(x^{\prime}\right)\right)$, for all $x^{\prime} \in U_{x}$. But $F$ is $A$-direct at $x$, so there is a neighborhood of $x$ where $\operatorname{Im} D F(a) \simeq \operatorname{Im} D F(b)$, for $a, b$ in this neighborhood. Hence the $\operatorname{Im} T_{z}$ for $z$ in this neighborhood are all $A$-isomorphic to a fixed $A$-module $P$. Call $h_{z}: \operatorname{Im} T_{z} \rightarrow P$ these $A$-isomorphisms. For every $x \in \operatorname{Im} F, x=F(x)$, and $U_{x}, V_{x}$ may be chosen so that $u_{x}\left(U_{x}\right)=$ $v_{x}\left(V_{x}\right)$. Then $v_{x}: V_{x} \cap \operatorname{Im} T_{x} \rightarrow v_{x}\left(V_{x}\right) \cap \operatorname{Im} F$ is a bijection: it is one to one over all of $V_{x}$, and if $v_{x}(z) \in \operatorname{Im} F$, say $v_{x}(z)=u_{x}\left(z^{\prime}\right)$,

$$
v_{x}(z)=F v_{x}(z)=F u_{x}\left(z^{\prime}\right)=v_{x} T_{x} u_{x}^{-1}\left(u_{x}\left(z^{\prime}\right)\right)=v_{x}\left(T_{x}\left(z^{\prime}\right)\right)
$$

so $v_{x}(z) \in v_{x}\left(V_{x} \cap \operatorname{Im} T_{x}\right)$.

Now define the chart near $x \in \operatorname{Im} F:\left(v_{x}\left(V_{x}\right) \cap \operatorname{Im} F, h_{x} v_{x}^{-1}\right)$. These charts are compatible. To see this, suppose

$$
U_{x y}=v_{x}\left(V_{x}\right) \cap v_{y}\left(V_{y}\right) \cap \operatorname{Im} F \neq \varnothing
$$

we then have

$$
\left(h_{y} v_{y}^{-1}\right)\left(h_{x} v_{x}^{-1}\right)^{-1}: h_{x} v_{x}^{-1}\left(U_{x y}\right) \rightarrow h_{y} v_{y}^{-1}\left(U_{x y}\right) .
$$

But $\left(h_{y} v_{y}^{-1}\right)\left(h_{x} v_{x}^{-1}\right)^{-1}=h_{y} v_{y}^{-1} v_{x} h_{x}^{-1}$ is holomorphic. The same goes for the other composition. The tangent space $T_{x}(\operatorname{Im} F)$ is given by

$$
\begin{gathered}
\operatorname{Im}\left(D v_{x}(0) h_{x}^{-1}\right)=D v_{x}(0)\left(\operatorname{Im} T_{x}\right)=\operatorname{Im}\left(D v_{x}(0) T_{x}\right)=\operatorname{Im} D\left(v_{x} T_{x}\right)(0) \\
=\operatorname{Im} D\left(F u_{x}\right)(0)=\operatorname{Im}\left(D F\left(u_{x}(0)\right) D u_{x}(0)\right)=\operatorname{Im} D F(x) .
\end{gathered}
$$

3. $A_{M}$ as an analytic manifold. Here we will apply the results in the preceding paragraph to Taylor's $A_{M}$ [7] where $M$ is a closed submanifold of an open set of $\mathbf{C}^{n}$.

For $a \in A^{n}$, let $\hat{a}$ denote the function $A^{\prime} \rightarrow \mathbf{C}^{n}$ defined by $\hat{a}(\gamma)=$ $\left(\gamma\left(a_{1}\right), \ldots, \gamma\left(a_{n}\right)\right)$ for all $\gamma \in A^{\prime}$. Note that with the supremum norm in both $A^{n}$ and $\mathbf{C}^{n},|\hat{a}(\gamma)| \leq\|\gamma\|\|a\|$. We will sometimes write $\phi^{n}$ for $\phi \times \cdots \times \phi$. We denote by $\theta_{a}$ the classical holomorphic functional calculus of Arens and Calderón [1]. All other functional calculus morphisms and their restrictions will be denoted by $\nu$.

We will need the following lemma.

LEMMA 3.1. Let $W$ be an open subset of $\mathbf{C}^{n}$. Then $A_{W}$ is an open subset of $A^{n}$. 
Proof. Let $a \in A_{W}$, and $f \in \mathcal{O}(X, W)$ such that $a=\nu(f)$. Since $f(X)$ is a compact subset of $W$, there is an $\varepsilon>0$ such that for every $\phi \in X$, the polydisc $\left\{z \in \mathbf{C}^{n}:|f(\phi)-z|<\varepsilon\right\}$ is contained in $W$. Now let $U=\{b \in$ $\left.A^{n}:\|a-b\|<\varepsilon\right\} . \hat{b}(X) \subseteq W$, because

$$
|f(\phi)-\hat{b}(\phi)|=|\widehat{a-b}(\phi)| \leq\|a-b\|<\varepsilon .
$$

Then $\hat{b}^{-1}(W)$ is a neighborhood of $X$ in $A^{\prime}$, so $\hat{b} \in \mathcal{O}(X, W)$, and $b \in A_{W}$.

The sets $A_{W}$, with $W$ open, are now appropriate domains for holomorphic functions. We will need to lift holomorphic functions in $\mathbf{C}^{n}$ to holomorphic functions in $A^{n}$. This will be done as follows. Let $h$ : $W \rightarrow \mathbf{C}^{m}$ be holomorphic, and define $A_{h}: A_{W} \rightarrow A^{m}$ by $A_{h}(a)=\nu(h \circ f)$, if $a=\nu(f)$.

LEMMA 3.2. $A_{h}$ is a well-defined holomorphic function. For all $a=\nu(f)$ $\in A_{W}, D A_{h}(a)$ is an A-module homomorphism given by $\nu(D h(f))$.

Proof. First, we will see that $\nu(f)=\nu(g)$ implies $\nu(h \circ f)=\nu(h \circ g)$.

For this, let $b_{1}, \ldots, b_{k} \in A$ be elements that finitely determine $f$ and $g$, in other words, there is an open set $\Omega$ in $C^{k}$ and there are $F$ and $G$ in $\mathcal{O}(\Omega, W)$ such that the following diagram commutes

$$
\begin{array}{cccc}
\hat{b}^{-1}(\Omega) & \stackrel{f \text { resp. } g)}{\rightarrow} \quad W & \stackrel{h}{\rightarrow} & \mathbf{C}^{m} \\
\hat{b} \downarrow & >_{F(\text { resp. } G)} & & \\
\Omega & &
\end{array}
$$

$\nu(f)=\nu(g)$ means that $\theta_{b}(F)=\theta_{b}(G)$, so $\operatorname{sp}\left(\theta_{b}(F)\right)=\operatorname{sp}\left(\theta_{b}(G)\right) \subseteq W$. Since $h \in \mathcal{O}\left(W, \mathbf{C}^{m}\right)$, we may write $\theta_{\theta_{b}(F)}(h)=\theta_{\theta_{b}(G)}(h)$. Then $h(F(b))=$ $h(G(b))$, so $\theta_{b}(h \circ F)=\theta_{b}(h \circ G)$ and $\nu(h \circ f)=\nu(h \circ g)$.

To prove that $A_{n}$ is holomorphic, let $a \in A_{W}$, and $b \in A^{n}$. It will be enough to prove the existence of

$$
\frac{\partial A_{h}}{\partial b}(a)=\lim _{\lambda \rightarrow 0} \frac{1}{\lambda}\left[A_{h}(a+\lambda b)-A_{h}(a)\right] .
$$

Let $a=\nu(f), \quad b=\nu(g)$. Then $a+\lambda b=\nu(f+\lambda g)$, and (1) is $\lim _{\lambda \rightarrow 0} \lambda^{-1}[\nu(h \circ(f+\lambda g)-h \circ f)]$. Since the functional calculus is continuous, the limit (1) will exist if $\lim _{\lambda \rightarrow 0} \lambda^{-1}[h \circ(f+\lambda g)-h \circ f]$ exists in $\mathcal{O}\left(X, C^{m}\right)$. We must see that $\lambda^{-1}[h \circ(f+\lambda g)-h \circ f]$ converges uniformly over a neighborhood of $X$ as $\lambda \rightarrow 0$. For this, set $\varepsilon>0$, and if $\lambda \in C$ with $|\lambda|<\varepsilon$ and $\gamma \in X$, let

$S(\lambda, \gamma)= \begin{cases}\frac{1}{\lambda}[h(f(\gamma)+\lambda g(\gamma))-h(f(\gamma))]-\frac{\partial h}{\partial g(\gamma)} f(\gamma), & \text { if } \lambda \neq 0 . \\ 0 & \text { if } \lambda=0 .\end{cases}$ 
$h$ is holomorphic, so $\lim _{\lambda \rightarrow 0} S(\lambda, \gamma)=0$ for each $\gamma \in X$. Then there are $\delta_{\gamma}>0$ and neighborhoods $V_{\gamma}$ of $\gamma$ such that $|S(\lambda, \phi)|<\varepsilon$ for $\lambda \in \mathbf{C}$ with $|\lambda|<\delta_{\gamma}$ and all $\phi \in V_{\gamma}$. Being $X$ compact, there are $\gamma_{1}, \ldots, \gamma_{p} \in X$ such that $V_{\gamma_{i}}, i=1, \ldots, p$, cover $X$. Let $\delta=\min \left\{\delta_{\gamma_{i}}: 1 \leq i \leq p\right\}$, and $V=$ $\bigcup_{i=1}^{p} V_{\gamma_{i}}$. Then for all $\lambda \in C$ with $|\lambda|<\delta$ and all $\gamma \in V, S(\lambda, \gamma)<\varepsilon$, so $A_{h}$ is holomorphic. We shall denote the limit of $\lambda^{-1}[h \circ(f+\lambda g)-h \circ f]$ as $\lambda \rightarrow 0$, by $\operatorname{Dh}(f)(g)$.

$D A_{h}(a)$ is more than just a linear morphism. It is $A$-linear. To prove this we must show that the diagram

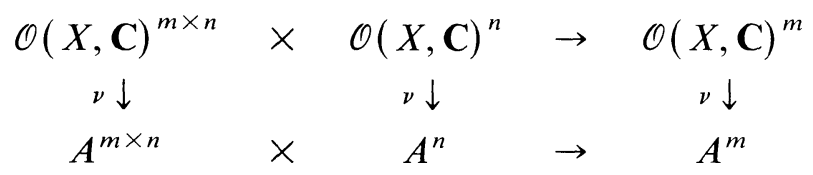

commutes.

Here the horizontal arrows indicate matrix multiplication.

As all the arrows are continuous, and $P(\hat{A})^{k}$ is dense in $\mathcal{O}(X, \mathbf{C})^{k}$ for all $k$, where $P(\hat{A})$ is the algebra of polynomials in Gelfand transforms of elements of $A$, it will be enough to show that $\nu(p \cdot q)=\nu(p) \cdot \nu(q)$, where $p_{i j}, q_{j} \in P(\hat{A})$. Let

$$
\begin{aligned}
& p_{i j}=\sum_{(k)} \widehat{a^{i j}}(k), \quad \text { where } \widehat{a^{i j}}(k)=\widehat{a}^{k^{k} k_{1}} \cdots{\widehat{a^{i j}}}_{k_{r}}^{k_{r}} \\
& q_{j}=\sum_{\left(k^{\prime}\right)} \widehat{a^{j}}\left(k^{\prime}\right), \quad \text { where } \widehat{a^{j}}\left(k^{\prime}\right)=\widehat{a^{j} k_{1}^{\prime}} \cdots \widehat{a^{j} k_{s}^{\prime}} . \\
& \nu(p \cdot q)=\nu\left(\sum_{j=1}^{n} p_{1 j} q_{j}, \ldots, \sum_{j=1}^{n} p_{m j} q_{j}\right) \\
& =\nu\left(\sum_{j=1}^{n} \sum_{(k)} \widehat{a^{1 j}}(k) \sum_{\left(k^{\prime}\right)} \widehat{a^{j}}\left(k^{\prime}\right), \ldots, \sum_{j=1}^{n} \sum_{(k)} \widehat{a^{m j}}(k) \underset{\left(k^{\prime}\right)}{ } \widehat{a^{j}}\left(k^{\prime}\right)\right) \\
& =\left(\sum_{j=1}^{n} \sum_{(k)} a^{1 j}(k) \sum_{\left(k^{\prime}\right)} a^{j}\left(k^{\prime}\right), \ldots, \sum_{j=1}^{n} \sum_{(k)} a^{m j}(k) \sum_{\left(k^{\prime}\right)} a^{j}\left(k^{\prime}\right)\right) .
\end{aligned}
$$

On the other hand,

$$
\nu(p) \cdot \nu(q)=\left(\sum_{j=1}^{n} \nu(p)_{1 j} \nu(q)_{j}, \ldots, \sum_{j=1}^{n} \nu(p)_{m j} \nu(q)_{j}\right) .
$$

But

$$
\nu(p)_{l j}=\nu\left(p_{l j}\right)=\nu\left(\sum_{(k)} \widehat{a^{l j}}(k)\right)=\sum_{(k)} a^{l j}(k),
$$


and

$$
\nu(q)_{j}=\nu\left(q_{j}\right)=\nu\left(\sum_{\left(k^{\prime}\right)} \widehat{a^{j}}\left(k^{\prime}\right)\right)=\sum_{\left(k^{\prime}\right)} a^{j}\left(k^{\prime}\right)
$$

So

$$
(2)=\left(\sum_{j=1}^{n} \sum_{(k)} a^{1 j}(k) \sum_{\left(k^{\prime}\right)} a^{j}\left(k^{\prime}\right), \ldots, \sum_{j=1}^{n} \sum_{(k)} a^{m j}(k) \sum_{\left(k^{\prime}\right)} a^{j}\left(k^{\prime}\right)\right)=\nu(p \cdot q) \text {. }
$$

Then

$$
D A_{h}(a)(b)=\nu(D h(f)(g))=\nu(D h(f)) \cdot \nu(g)=\nu(D h(f))(b) .
$$

So that $D A_{h}(a)=\nu(D h(f)) \in A^{m \times n}$ is an $A$-module morphism, for all $a \in A_{W}$.

Note that $A_{h}$ could have been well-defined by putting $A_{h}(a)=$ $\nu(h \circ \hat{a})$, but this definition will not do for our later purposes.

Now let $M$ be a closed submanifold of an open set of $\mathbf{C}^{n}$, of dimension $k$. We recall that by [3; Ch. VIII, C] there is an open neighborhood $W$ of $M$ and an holomorphic retraction $r: W \rightarrow M$. Hence we also have $A_{r}: A_{W} \rightarrow A_{M}$, the image of $A_{r}$ being contained in $A_{M}$ because $r \circ f \in \mathcal{O}(X, M)$ for all $f \in \mathcal{O}(X, W)$. Of course the image of $A_{r}$ is exactly $A_{M}$, for if $a \in A_{M}$, then $A_{r}(a)=\nu(r \circ f)$ where $f \in \mathcal{O}(X, M)$ so $r \circ f=f$, and $A_{r}(a)=\nu(r \circ f)=\nu(f)=a \in \operatorname{Im} A_{r}$. Now we obtain our main theorem.

THEOREM 3. If $M$ is a closed submanifold of an open set of $\mathbf{C}^{n}$, of dimension $k$, then $A_{M}$ is a Banach manifold modeled on projective A-modules of rank $k$.

Proof. By Theorem 2, it will clearly be enough to verify that $A_{r}$ is $A$-direct at $a$ for all $a$ in a neighborhood of $A_{M}$.

Since $r$ is a retraction, $\operatorname{Dr}(r(z)) \circ \operatorname{Dr}(z)=\operatorname{Dr}(z)$ for all $z \in W$. Therefore $\operatorname{Im} \operatorname{Dr}(z) \subseteq \operatorname{Im} \operatorname{Dr}(r(z))$, but the rank of the matrix $\operatorname{Dr}(z)$ is at least that of $\operatorname{Dr}(r(z))$ for $z$ near $r(z)$, so that actually $\operatorname{Im} \operatorname{Dr}(z)=$ $\operatorname{Im} \operatorname{Dr}(r(z))$ for $z$ in an open neighborhood of $M$. This means that $\operatorname{dim} \operatorname{Im} \operatorname{Dr}(z)=k$, and $\operatorname{dim} \operatorname{Ker} \operatorname{Dr}(z)=n-k$ near $M . \mathbf{C}^{n}$ can be written as the direct sum

$$
\mathbf{C}^{n}=\operatorname{Im} \operatorname{Dr}(r(z)) \oplus \operatorname{Ker} \operatorname{Dr}(r(z))=\operatorname{Im} \operatorname{Dr}(z) \oplus \operatorname{Ker} \operatorname{Dr}(r(z)) .
$$

Because of the continuity of $\operatorname{Dr}$, we may also write $\mathbf{C}^{n}=\operatorname{Im} \operatorname{Dr}(z) \oplus$ $\operatorname{Ker} \operatorname{Dr}(z)$, for $z$ near $M$. Note also that $\operatorname{Dr}(r(z)) \mid \operatorname{Im} \operatorname{Dr}(r(z))$ is the identity, so that $\operatorname{Dr}(z) \mid \operatorname{Im} \operatorname{Dr}(z)$ is an automorphism of $\operatorname{Im} \operatorname{Dr}(z)$ near $M$. We may suppose the neighborhood of $M$ where all this is true to be $W$; 
just discard the old $W$. For all $z \in W$,

$$
\alpha_{z}=\left[\begin{array}{ccc}
\operatorname{Dr}(z) & \vdots & 0 \\
\cdots & \vdots & \vdots \\
0 & \vdots
\end{array}\right]:\left[\begin{array}{c}
\operatorname{Im} \operatorname{Dr}(z) \\
\operatorname{Ker} \operatorname{Dr}(z)
\end{array}\right] \rightarrow\left[\begin{array}{c}
\operatorname{Im} \operatorname{Dr}(z) \\
\operatorname{Ker} \operatorname{Dr}(z)
\end{array}\right],
$$

is an automorphism of $\mathbf{C}^{n}$. Define $\alpha: W \rightarrow \mathrm{GL}_{n}(\mathbf{C})$ by $\alpha(z)=$ the matrix of $\alpha_{z}$ in the canonical basis of $\mathbf{C}^{n}$. We will show that $\alpha$ is an holomorphic function. For this, let $z_{0} \in W$. There is a neighborhood $U$ of $z_{0}$ and there are holomorphic functions $v_{i}: U \rightarrow \mathbf{C}^{n}, \quad 1 \leq i \leq n$, such that $v_{1}(z), \ldots, v_{k}(z)$ is a basis of $\operatorname{Im} \operatorname{Dr}(z)$ and $v_{k+1}(z), \ldots, v_{n}(z)$ is a basis of $\operatorname{Ker} \operatorname{Dr}(z)$ for all $z \in U$. Let $\beta_{z} \in \mathbf{C}^{k \times k}$ be the matrix of $\operatorname{Dr}(z) \mid \operatorname{Im} \operatorname{Dr}(z)$ in the basis $v_{1}(z), \ldots, v_{k}(z)$ and let $c(z)$ be the matrix which changes the canonical basis of $\mathbf{C}^{n}$ to $v_{1}(z), \ldots, v_{n}(z)$. Then

$$
\alpha(z)=c(z)^{-1} \cdot\left[\begin{array}{ccc}
\beta_{z} & \vdots & 0 \\
\vdots & \vdots & I
\end{array}\right] \cdot c(z)
$$

and it will be enough to verify that $\beta_{z}$ is an holomorphic function of $z$ in $U$, but this follows from the equations

$$
\operatorname{Dr}(z)\left(v_{i}(z)\right)_{t}=\sum_{s=1}^{k} \beta_{z_{t s}} v_{i}(z)_{s}, \quad i \leq i, t \leq k .
$$

We therefore have $A_{\alpha}: A_{W} \rightarrow A_{\mathrm{GL}_{n}(\mathrm{C})}=\mathrm{GL}_{n}(A)$. But

$$
\left.A_{\alpha}(x)\right|_{\operatorname{Im} D A_{r}(x)}=\left.D A_{r}(x)\right|_{\operatorname{Im} D A_{r}(x)}
$$

for all $x \in A_{W}$. To see this, let $b=\nu(\operatorname{Dr}(g)(h)) \in \operatorname{Im} D A_{r}(x)$, where $x=\nu(g)$. Now $A_{\alpha}(x)(b)=\nu(\alpha \circ g) \cdot \nu(\operatorname{Dr}(g)(h))=\nu(\alpha \circ g \cdot \operatorname{Dr}(g)(h))$, but for all $\gamma$ near $X$,

$$
\left.\alpha(g(\gamma))\right|_{\operatorname{Im} \operatorname{Dr}(g(\gamma))}=\left.\operatorname{Dr}(g(\gamma))\right|_{\operatorname{Im} \operatorname{Dr}(g(\gamma))},
$$

so

$$
\begin{aligned}
A_{\alpha}(x)(b) & =\nu(\operatorname{Dr}(g) \cdot \operatorname{Dr}(g)(h)) \\
& =\nu(\operatorname{Dr}(g)) \cdot \nu(\operatorname{Dr}(g)(h))=D A_{r}(x)(b) .
\end{aligned}
$$

Then

$$
\left.D A_{r}(x)\right|_{\operatorname{Im} D A_{r}(x)}: \operatorname{Im} D A_{r}(x) \rightarrow \operatorname{Im} D A_{r}(x) \text { is an automorphism. }
$$

We prove that $A^{n}=\operatorname{Im} D A_{r}(x) \oplus \operatorname{Ker} D A_{r}(x)$ for all $x \in A_{W}$ :

$$
0=\operatorname{Ker}\left(\left.D A_{r}(x)\right|_{\operatorname{Im} D A_{r}(x)}\right)=\operatorname{Im} D A_{r}(x) \cap \operatorname{Ker} D A_{r}(x) .
$$

If $c \in A^{n}$, there exists $b \in \operatorname{Im} D A_{r}(x)$ such that $D A_{r}(x)(b)=D A_{r}(x)(c)$. Then $c=b+(c-b)$, with $b \in \operatorname{Im} D A_{r}(x)$ and $c-b \in \operatorname{Ker} D A_{r}(x)$. $\operatorname{Ker} D A_{r}(x)$ is closed, so the direct sum is topological. 
We now know that $\operatorname{Im} D A_{r}(x)$ is a projective $A$-module.

We shall see that its rank is $k$.

First we must prove that for all $x \in A_{W}$ and $\phi \in X$,

$$
\phi^{n}\left(\operatorname{Im} D A_{r}(x)\right)=\operatorname{Im} \operatorname{Dr}\left(\phi^{n}(x)\right)
$$

and

Take

$$
\phi^{n}\left(\operatorname{Ker} D A_{r}(x)\right)=\operatorname{Ker} \operatorname{Dr}\left(\phi^{n}(x)\right) \text {. }
$$

$$
\begin{aligned}
D A_{r}(x)(b) & \in \operatorname{Im} D A_{r}(x) \cdot \phi^{n}\left(D A_{r}(x)(b)\right)=\widehat{\nu(\operatorname{Dr}(\hat{x})(\hat{b}))}(\phi) \\
& =(\operatorname{Dr}(\hat{x})(\hat{b}))(\phi)=\operatorname{Dr}\left(\phi^{n}(x)\right)\left(\phi^{n}(b)\right) \in \operatorname{Im} \operatorname{Dr}\left(\phi^{n}(x)\right) .
\end{aligned}
$$

Now take $b \in \operatorname{Ker} D A_{r}(x)$.

$$
\operatorname{Dr}\left(\phi^{n}(x)\right)\left(\phi^{n}(b)\right)=\phi^{n}\left(D A_{r}(x)(b)\right)=\phi^{n}(0)=0,
$$

so $\phi^{n}(b) \in \operatorname{Ker} \operatorname{Dr}\left(\phi^{n}(x)\right)$, and we have proven both left-to-right inclusions. We have $A^{n}=\operatorname{Im} D A_{r}(x) \oplus \operatorname{Ker} D A_{r}(x)$, and $\phi^{n}$ is surjective, so

$$
\mathbf{C}^{n}=\phi^{n}\left(\operatorname{Im} D A_{r}(x)\right)+\phi^{n}\left(\operatorname{Ker} D A_{r}(x)\right),
$$

but because of the inclusions we have just proven, this sum is direct. Then

$$
\begin{aligned}
\mathbf{C}^{n} & =\phi^{n}\left(\operatorname{Im} D A_{r}(x)\right) \oplus \phi^{n}\left(\operatorname{Ker} D A_{r}(x)\right) \\
& =\operatorname{Im} \operatorname{Dr}\left(\phi^{n}(x)\right) \oplus \operatorname{Ker} \operatorname{Dr}\left(\phi^{n}(x)\right),
\end{aligned}
$$

so the inclusions are actually equalities.

Now let $x \in A_{W}, P=\operatorname{Im} D A_{r}(x), Q=\operatorname{Ker} D A_{r}(x)$, and $\phi \in X$. Then $\mathrm{rk}_{\phi} P=\mathrm{rk}_{A_{\phi}} P_{\phi}=\mathrm{rk}_{A_{\phi}}\left(A_{\phi} \otimes_{A} P\right)$ is, by Nakayama's Lemma the same as $\operatorname{dim}_{\mathbf{C}}\left[\left(A_{\phi} \otimes_{A} P\right) \otimes_{A_{\phi}} \mathrm{C}\right]$, when $\mathrm{C}$ (and also $\left.\phi^{n}(P)\right)$ has the $A_{\phi}$-module structure induced by $\phi$. We then have the $A_{\phi}$-module morphism

$$
\begin{gathered}
q:\left(A_{\phi} \otimes_{A} P\right) \otimes_{A_{\phi}} \mathrm{C} \rightarrow \phi^{n}(P) ; \\
q\left(\sum_{j}\left(\sum_{i} \frac{a_{i j}}{b_{i j}} \otimes p_{i j}\right) \otimes \lambda_{j}\right)=\sum_{j} \sum_{i} \lambda_{j} \frac{\phi\left(a_{i j}\right)}{\phi\left(b_{i j}\right)} \phi^{n}\left(p_{i j}\right) .
\end{gathered}
$$

Let $v_{1}, \ldots, v_{k}$ has a basis for $\phi^{n}(P)=\operatorname{Im} \operatorname{Dr}\left(\phi^{n}(x)\right)$, and let $b_{1}, \ldots, b_{k}$ $\in P$ such that $\phi^{n}\left(b_{i}\right)=v_{i}$ for $i=1, \ldots, k$. Then $\left(1 / 1 \otimes b_{i}\right) \otimes 1, i=$ $1, \ldots, k$, are C-linearly independent: if $0=\sum_{i=1}^{k} \lambda_{i}\left(1 / 1 \otimes b_{i}\right) \otimes 1$, then

$$
0=q(0)=\sum_{i=1}^{k} \lambda_{i} \phi^{n}\left(b_{i}\right)=\sum_{i=1}^{k} \lambda_{i} v_{i}
$$

and $\lambda_{i}=0$ for $i=1, \ldots, k$.

Therefore $\mathrm{rk}_{\phi} P=\operatorname{dim}_{\mathbf{C}}\left[\left(A_{\phi} \otimes_{A} P\right) \otimes_{A_{\phi}} \mathbf{C}\right] \geq k$.

In a similar manner, and since $\phi^{n}(Q)=\operatorname{Ker} \operatorname{Dr}\left(\phi^{n}(x)\right), \operatorname{rk}_{\phi} Q \geq$ $n-k$. But $\mathrm{rk}_{\phi} P+\mathrm{rk}_{\phi} Q=n$, so $\operatorname{rk}_{\phi} P=k \forall \phi \in X$. Then rk $P=k$. 
To complete our proof, let $a \in A_{M}$ and write:

$$
D A_{r}(x)=\left[\begin{array}{ll}
P(x) & Q(x) \\
R(x) & S(x)
\end{array}\right]:\left[\begin{array}{c}
\operatorname{Im} D A_{r}(a) \\
\operatorname{Ker} D A_{r}(a)
\end{array}\right] \rightarrow\left[\begin{array}{c}
\operatorname{Im} D A_{r}(a) \\
\operatorname{Ker} D A_{r}(a)
\end{array}\right] .
$$

Since $D A_{r}(a)$ is an indempotent, $\left.D A_{r}(a)\right|_{\operatorname{Im} D A_{r}(a)}$ is the identity, and $P(a)=I$. But $\operatorname{Im} D A_{r}(a)$ is a Banach space, so by the continuity of $P$, $P(x)$ is an automorphism of $\operatorname{Im} D A_{r}(a)$ for all $x$ in a neighborhood $U$ of $a$.

We have then verified conditions (iv) of Theorem 1 for all $x \in U$. Therefore, $A_{r}$ is $A$-direct at $x$ for all $x$ in a neigborhood of $A_{M}$.

Observe that the tangent space $T_{a}\left(A_{M}\right)$ at $a$ is $\operatorname{Im} D A_{r}(a)$. These are of course projective $A$-modules of rank $k$, but they need not be isomorphic on different connected components of $A_{M}$. In fact, some of these modules may be free while others may not.

Now consider for any Banach algebra $A$, the category $M(A)$ whose objects are analytic manifolds modeled on projective $A$-modules, with morphisms holomorphic functions whose differentials are $A$-module morphism, and the ordinary composition. Let $\underline{M}$ be the category of closed analytic submanifolds of open subsets of finite products of $\mathbf{C}$. Then we have:

Proposition 3.3. $A_{(\cdot)}$ is a covariant functor from $\underline{M}$ to $\underline{M(A)}$.

Proof. $A_{M}$ is defined for every object in $\underline{M}$ and is an object of $M(A)$, by Theorem 3. Now let $M$ and $N$ be two objects of $\underline{M}$ and $h: M \rightarrow \overline{\rightarrow \text { an }}$ holomorphic function. $h$ can be extended to an open neighborhood $W$ of $M$ for example by $h \circ r$. If $\bar{h}$ is such an extension, then we can define $A_{\bar{h}}$ as before Lemma 3.2. Now define $A_{h}$ to be the restriction of $A_{\bar{h}}$ to $A_{M}$, for any extension $\bar{h}$ of $h$. Obviously, $\operatorname{Im} A_{h}=A_{\bar{h}}\left(A_{M}\right) \subseteq A_{N}$, and if $h_{1}$ and $h_{2}$ are two extensions of $h$, and $a \in A_{M}, a=\nu(f)$ with $f \in \mathcal{O}(X, M)$, then

$$
A_{h_{1}}(a)=\nu\left(h_{1} \circ f\right)=\nu(h \circ f)=\nu\left(h_{2} \circ f\right)=A_{h_{2}}(a) \text {, }
$$

so $A_{h}$ is well defined. The rest of the Proposition is easily verified.

There are many holomorphic functions in $A^{n}$ whose differentials are $A$-module morphisms, but which are not of the form $A_{h}$ for any $h$. As an example, take $a \in A$ such that there are $x \in A$, and $\phi, \psi \in X$ with $\phi(x)=\psi(x) \neq 0$ and $\phi(a) \neq \psi(a)$; and consider $L_{a}: A \rightarrow A$ defined by $L_{a}(y)=a y . L_{a}$ is $A$-linear, but $L_{a} \neq A_{h}$ for all $h$ : if $L_{a}$ were $A_{h}, a x=$ $L_{a}(x)=A_{h}(x)=\nu(h \circ \hat{x})$, so over $X, \hat{a} \hat{x}=h \circ \hat{x}$, and then

$$
\phi(a) \cdot \phi(x)=h(\phi(x))=h(\psi(x))=\psi(a) \psi(x) .
$$

Hence, $\phi(a)=\psi(a)$, contrary to our assumptions.

Finally, we wish to compare $A_{M}$ and $A^{M}$. 
Proposition 3.4. $A^{M}=A_{M}+\operatorname{Rad}(A)^{n}$.

Proof. Let $\mathscr{N}=\left\{f \in \mathcal{O}(X, \mathbf{C}):\left.f\right|_{X}=0\right\}$. Then $\nu(\mathscr{N})=\operatorname{Rad}(A)$ : if $f \in \mathscr{N}, \overline{\nu(f)}_{X}=\left.f\right|_{X}=0$, so $\nu(\mathscr{N}) \subseteq \operatorname{Rad}(A)$; on the other hand, if $a \in \operatorname{Rad}(A), a=\nu(\hat{a})$ with $\hat{a} \mid X=0$. We identify also $\operatorname{Rad}(A)^{n}$ with $\nu\left(\mathscr{N}^{n}\right)$. Note that $A^{M} \subseteq A_{W}$, for if $\hat{a}(X)=\operatorname{sp}(a) \subseteq M$, then $\hat{a} \in$ $\mathcal{O}(X, W)$. Now take $a \in A^{M}$, and put $a=A_{r}(a)+\left(a-A_{r}(a)\right) . A_{r}(a) \in$ $A_{M}$, and

$$
a-A_{r}(a)=\nu(\hat{a})-\nu(r \circ \hat{a})=\nu(\hat{a}-r \circ \hat{a}) \in \operatorname{Rad}(A)^{n},
$$

because $\hat{a}-r \circ \hat{a} \in \mathscr{N}^{n}$. For the other inclusion, let $b \in A_{M}$ and $c \in$ $\operatorname{Rad}(A)^{n} . c=\nu(g)$, with $g \in \mathscr{N}^{n}$. Then

$$
\begin{aligned}
\operatorname{sp}(b+c) & =\widehat{b+c}(X)=(\hat{b}+\widehat{\nu(g)})(X) \\
& =(\hat{b}+g)(X)=\hat{b}(X)=\operatorname{sp}(b) \subseteq M .
\end{aligned}
$$

Corollary 3.5. $A^{M}$ and $A_{M}$ have the same homotopy type. If $A$ is semisimple, then $A^{M}=A_{M}$. (See also [7; 2.8].)

Proof. Let $\iota: A_{M} \rightarrow A^{M}$ denote the inclusion. $A_{r} \circ \iota$ is the identity on $A_{M}$ and it is easily verified that $\iota \circ A_{r}$ is homotopic to the identity on $A^{M}$.

4. An example. We wish to consider briefly an example of a spectral set. Suppose $A$ is semisimple, and the manifold $M$ is given as the zero set of a holomorphic function

$$
W \stackrel{F}{\rightarrow} \mathbf{C}^{k} .
$$

It has been established in the last paragraph that $A_{M}$ is a Banach manifold. This would have been a much simpler matter in this particular case, but a bit more can be said. Lift $F$ to an analytic function

$$
A_{W} \stackrel{A_{F}}{\rightarrow} A^{k}
$$

and the zero set of $A_{F}$ is exactly $A_{M}$. To see this, let $a \in A_{M}$; then $a=\nu(f)$ with $f \in \mathcal{O}(X, M)$, and $A_{F}(a)=\nu(F \circ f)=\nu(0)=0$, so $a \in$ $A_{F}^{-1}(0)$. Now if $A_{F}(a)=0, \nu(F \circ \hat{a})=0$ and $F \circ \hat{a}=0$ over $X$. Hence $F(\operatorname{sp}(a))=\{0\}$, and $\operatorname{sp}(a) \subset M$. We then have $A_{M} \subset A_{F}^{-1}(0) \subset A^{M}$, but since $A$ is semisimple, all three are the same.

Now take $W=\mathrm{GL}_{n}(\mathbf{C})$, and $G$ a Lie subgroup of $W$ which is the zero set of analytic functions, for instance an algebraic group. Then the corresponding zero set of the same functions in $\mathrm{GL}_{n}(A)$ is a Lie subgroup of $\mathrm{GL}_{n}(A)$.

It can in fact be shown that all Lie groups give rise to Banach Lie groups, and that these have tangent spaces which are free $A$-modules. 


\section{REFERENCES}

[1] (i)R. Arens and A. P. Calderón, Analytic functions of several Banach algebra elements, Ann. of Math., (2) 62 (1955), 204-216.

[2] I. Craw, $A$ condition equivalent to the continuity of characters on a Fréchet algebra, Proc. London Math. Soc., 22 (1971), 452-464.

[3] R. C. Gunning and H. Rossi, Analytic Functions of Several Complex Variables, Prentice Hall, Englewood Cliffs, N.J., 1965.

[4] A. Larotonda, Notas sobre variedades diferenciables, INMABB--CONICET, Bahía Blanca, 1980.

[5] E. Novodvorskii, Certain homotopical invariants of spaces of maximal ideals, Mat. Zametki, 1 (1967), 487-494.

[6] I. Raeburn, The relationship between a commutative Banach algebra and its maximal ideal space, J. Functional Anal., 25 (1977), 366-390.

[7] J. L. Taylor, Topological invariants of the maximal ideal space of a Banach algebra, Adv. in Math., 19 (1976), 149-206.

[8] _ _ Twisted Products of Banach Algebras and Third Čech Cohomology, in Springer Lecture Notes in Math., 575 (1977), 157-174.

Received March 8, 1984.

UNIVERSIDAD DE BUENOS AIRES

Pabellon I - CiUdad Universitaria

CAPITAL Federal (1428)

ARgentina 


\section{PACIFIC JOURNAL OF MATHEMATICS EDITORS}

\author{
V. S. VARADARAJAN (Managing Editor) \\ University of California \\ Los Angeles, CA 90024 \\ Charles R. DePrima \\ California Institute of Technology \\ Pasadena, CA 91125 \\ R. FINN \\ Stanford University \\ Stanford, CA 94305
}

\author{
HeRmanN FlaschKa \\ University of Arizona \\ Tucson, AZ 857.21
}

RAMESH A. GANGOLli

University of Washington

Seattle, WA 98195

ROBION KIRBY

University of California

Berkeley, CA 94720
C. C. MOORE

University of California

Berkeley, CA 94720

H. SAMELSON

Stanford University

Stanford, CA 94305

HAROLD STARK

University of California, San Diego

La Jolla, CA 92093

\section{ASSOCIATE EDITORS}
R. ARENS
E. F. BECKENBACH
B. H. NeumanN
F. WOLF
K. YoSHIDA
(1906-1982)

\section{SUPPORTING INSTITUTIONS}

\begin{abstract}
UNIVERSITY OF ARIZONA
UNIVERSITY OF BRITISH COLUMBIA

CALIFORNIA INSTITUTE OF TECHNOLOGY

UNIVERSITY OF CALIFORNIA

MONTANA STATE UNIVERSITY

UNIVERSITY OF NEVADA, RENO

NEW MEXICO STATE UNIVERSITY

OREGON STATE UNIVERSITY
\end{abstract}

\author{
UNIVERSITY OF OREGON \\ UNIVERSITY OF SOUTHERN CALIFORNIA \\ STANFORD UNIVERSITY \\ UNIVERSITY OF HAWAII \\ UNIVERSITY OF TOKYO \\ UNIVERSITY OF UTAH \\ WASHINGTON STATE UNIVERSITY \\ UNIVERSITY OF WASHINGTON
}

The Supporting Institutions listed above contribute to the cost of publication of this Journal, but they are not owners or publishers and have no responsibility for its content or policies.

Mathematical papers intended for publication in the Pacific Journal of Mathematics should be in typed form or offset-reproduced (not dittoed), double spaced with large margins. Please do not use built up fractions in the text of the manuscript. However, you may use them in the displayed equations. Underline Greek letters in red, German in green, and script in blue. The first paragraph must be capable of being used separately as a synopsis of the entire paper. In particular it should contain no bibliographic references. Please propose a heading for the odd numbered pages of less than 35 characters. Manuscripts, in triplicate, may be sent to any one of the editors. Please classify according to the scheme of Math. Reviews, Index to Vol. 39. Supply name and address of author to whom proofs should be sent. All other communications should be addressed to the managing editor, or Elaine Barth, University of California, Los Angeles, California 90024.

There are page-charges associated with articles appearing in the Pacific Journal of Mathematics. These charges are expected to be paid by the author's University, Government Agency or Company. If the author or authors do not have access to such Institutional support these charges are waived. Single authors will receive 50 free reprints; joint authors will receive a total of 100 free reprints. Additional copies may be obtained at cost in multiples of 50 .

The Pacific Journal of Mathematics is issued monthly as of January 1966. Regular subscription rate: $\$ 190.00$ a year (5 Vols., 10 issues). Special rate: $\$ 66.00$ a year to individual members of supporting institutions.

Subscriptions, orders for numbers issued in the last three calendar years, and changes of address should be sent to Pacific Journal of Mathematics, P.O. Box 969, Carmel Valley, CA 93924, U.S.A. Old back numbers obtainable from Kraus Periodicals Co., Route 100, Millwood, NY 10546.

The Pacific Journal of Mathematics at P.O. Box 969, Carmel Valley, CA 93924 (ISSN 0030-8730) publishes 5 volumes per year. Application to mail at Second-class postage rates is pending at Carmel Valley, California, and additional mailing offices. Postmaster: Send address changes to Pacific Journal of Mathematics, P.O. Box 969, Carmel Valley, CA 93924.

PUBLISHED BY PACIFIC JOURNAL OF MATHEMATICS, A NON-PROFIT CORPORATION

Copyright $\odot 1985$ by Pacific Journal of Mathematics 


\section{Pacific Journal of Mathematics}

Vol. 120, No. $2 \quad$ October, 1985

Philip Marshall Anselone and Mike Treuden, Regular operator

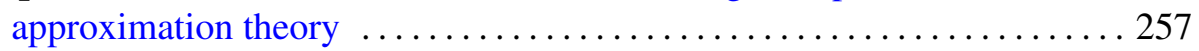

Giuseppe Baccella, Semiprime $\aleph-Q F 3$ rings $\ldots \ldots \ldots \ldots \ldots \ldots \ldots \ldots . \ldots \ldots$

Earl Robert Berkson and Thomas Alastair Gillespie, The generalized M.

Riesz theorem and transference $\ldots \ldots \ldots \ldots \ldots \ldots \ldots \ldots . \ldots 279$

Joachim Boidol, A Galois-correspondence for general locally compact

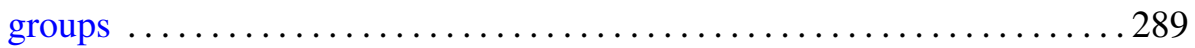

Joseph Eugene D'Atri, Josef Dorfmeister and Yan Da Zhao, The isotropy

representation for homogeneous Siegel domains ............... 295

C. Debiève, On Banach spaces having a Radon-Nikodým dual

Michael Aaron Freedman, Existence of strong solutions to singular

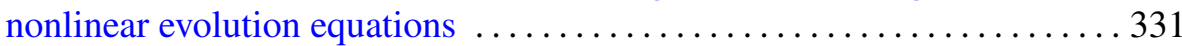

Francisco Jose Freniche, Grothendieck locally convex spaces of continuous

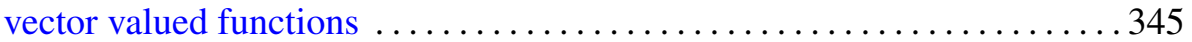

Hans-Peter Künzi and Peter Fletcher, Extension properties induced by complete quasi-uniformities . ............................ 357

Takaŝi Kusano, Charles Andrew Swanson and Hiroyuki Usami, Pairs of

positive solutions of quasilinear elliptic equations in exterior domains . . 385

Angel Rafael Larotonda and Ignacio Zalduendo, Spectral sets as Banach

manifolds

J. Martínez-Maurica and C. Pérez García, A new approach to the

Kreı̆n-Milman theorem

Christian Pommerenke, On the boundary continuity of conformal maps . . . 423

M. V. Subba Rao, Some Rogers-Ramanujan type partition theorems

Stephen Edwin Wilson, Bicontactual regular maps .........

Jaap C. S. P. van der Woude, Characterizations of (H)PI extensions

Kichoon Yang, Deformation of submanifolds of real projective space

Subhashis Nag, Errata: "On the holomorphy of maps from a complex to a

real manifold" 